\title{
Locating Faults on Untransposed, Meshed Transmission Networks Using a Limited Number of Synchrophasor Measurements
}

\author{
Sadegh Azizi, Student Member, IEEE, Majid Sanaye-Pasand, Senior Member, IEEE, and \\ Mario Paolone, Senior Member, IEEE
}

\begin{abstract}
The method of symmetrical components is not effective for fault location in the case of untransposed lines, due to potential couplings between the sequence circuits. This paper proposes a non-iterative algorithm in the phase-coordinates for wide-area fault location on untransposed transmission networks. In doing so, first, an improved two-terminal method is suggested to accurately locate faults on untransposed lines. Next, an algorithm is proposed to infer voltage and current phasors at the faulted line ends without direct measurements, by taking advantage of the data provided by phasor measurement units (PMUs). Accordingly, the adverse effect of close instrument transformers transients on the estimation accuracy is minimized. Being highly nonlinear in terms of fault distance and impedance, the fault equations are derived and made linear in this paper by defining six suitable auxiliary variables. The resulting system of equations is solved using the least-squares method to obtain three-phase voltages and currents at the faulted line ends. A main feature of the proposed algorithm is that it only requires a limited number of current and voltage synchrophasors. An additional advantage of the proposed algorithm is that the faulted line is not required to be known a-priori. The proposed algorithm is validated using extensive simulation studies on the New England 39-bus test system, accounting for different fault locations, types and resistances.
\end{abstract}

Index Terms-Fault location, least-squares method, short-circuit faults, synchrophasors, untransposed lines.

\section{INTRODUCTION}

$\mathbf{E}$ LECTRIC power networks are always prone to faults. Protection systems, and subsequent fault location processes are exploited to detect and isolate the smallest possible area including the faulted component, and restore the normal operating condition. In this respect, accurate fault location is necessary to improve the system reliability by reducing the outage and service restoration time [1], [2].

In general, conventional fault location methods can be classified into two major categories. The first one, which is more

Manuscript received April 28, 2015; revised August 16, 2015 and November 19, 2015; accepted December 17, 2015. This work was supported by the University of Tehran under Grant 8101064-1-08. Paper no. TPWRS-00589-2015.

S. Azizi and M. Sanaye-Pasand are with the School of Electrical and Computer Engineering, College of Engineering, University of Tehran, Tehran 14395515, Iran (e-mail: sadegh.azizi@ut.ac.ir; msanaye@ut.ac.ir).

M. Paolone is with the Distributed Electrical Systems Laboratory (DESL), École Polytechnique Fédérale de Lausanne (EPFL), Lausanne 1015, Switzerland (e-mail: mario.paolone@epfl.ch).

Color versions of one or more of the figures in this paper are available online at http://ieeexplore.ieee.org.

Digital Object Identifier 10.1109/TPWRS.2016.2517185 popular in real applications, utilizes the fundamental-frequency components of voltage and current signals [3]-[8]. The second category involves methods taking advantage of the fault-generated traveling waves [9]-[14]. Moreover, artificial intelligence has been used for fault location in a number of research works such as [15]-[19].

A common characteristic of conventional methods is the use of sufficiently independent information that might be inexact (or unavailable) during faults. Indeed, instrument transformers may lose their accuracy or, in extreme cases, their capability to correctly transduce post-fault signals for a transient period subsequent to the fault inception. This is more likely to happen, and lasts longer, for close instrument transformers to the fault point [1]. However, conventional methods formulate the problem based on the quantities measured at the faulted line ends, which are also the closest ones to the fault. In this context, wide-area fault location is a viable alternative that provides the possibility of using more reliable measurements, which, in general, are not close to the fault [20]-[36]. Among these methods, the impedance-based ones have been essentially developed for transposed transmission networks, or lower voltage levels where the line shunt admittances can be neglected [20]-[33]. Accordingly, none of such methods are suitable for transmission networks comprised of untransposed and/or not completely transposed lines. In practice, however, many transmission lines are left untransposed along their routes due to technical and/or economic reasons [37]-[40].

To overcome the above-mentioned deficiencies, a general fault location algorithm is proposed in this paper. This algorithm is developed so as to take advantage of the phasor measurement units (PMUs) already available in the system. The reason is because it is not rational to expect that PMUs are installed merely for maximizing the performance of wide-area fault location. An improved two-terminal method is proposed to pinpoint the fault on an untransposed transmission line. Next, the concept of wide-area fault location provided by synchrophasors is used to infer voltage and current phasors at the faulted line ends without direct measurements. To do so, the nonlinear three-phase fault equations relating the available synchrophasors are derived. These equations are made linear by defining six suitable auxiliary variables to guarantee a closed-form solution of the problem. By solving the resulting system of equations, three-phase voltage and current phasors at the faulted line ends are readily obtained. Once the current and voltage synchrophasors have been rigorously obtained, any 
suitable one- or two-terminal fault location methods can be applied for fault location. In this paper, the proposed two-terminal method has been applied to further improve the fault location accuracy on untransposed lines.

The salient features of the proposed algorithm can be outlined as follows:

- Use of a restricted number of synchrophasor measurements to pinpoint the fault location and type,

- Applicability to untransposed transmission networks,

- Capability of locating symmetrical/asymmetrical faults,

- Computational efficiency in addition to high accuracy.

The rest sections of this paper are organized as follows. Section II sets forth an accurate two-terminal fault location method for untransposed transmission lines. Afterwards, a technique is proposed to infer three-phase voltage and current phasors at the faulted line ends using the PMU data. These two techniques are then merged to derive the proposed wide-area fault location algorithm. Section III is devoted to performance evaluation of the proposed algorithm and the associated discussions. Finally, Section IV concludes the paper with final remarks.

\section{Proposed Algorithm}

In the first subsection, a two-terminal technique is proposed to locate generic faults (symmetrical and asymmetrical) on an untransposed transmission line. This method needs synchronized voltage and current phasors at the faulted line ends similar to other methods proposed in the literature. In the next subsection, an algorithm is set forth to use the data of a restricted number of PMUs for obtaining voltage and current phasors at the faulted line ends. This enables applying the proposed twoterminal method without direct measurement of the related input signals, which can be inexact due to measurement transformers transients.

\section{A. Accurate Fault Location on Untransposed Multi-Conductor Transmission Lines}

Equating the voltage at the fault point derived from both sides of the faulted line, gives three equations in terms of hyperbolic functions of $3 \times 3$ line parameter matrices and fault distance. In the transposed lines case, these equations are independent in the sequence domain and can be individually solved. However, in the case of untransposed lines, it is not possible to separate these equations and obtain a closed-form solution for the fault location since matrices multiplication does not commute [40]. This is why conventional two-terminal methods use some simplified assumptions to locate faults on untransposed transmission lines. Typical ones use the linear approximation of faulted line parameters and/or neglect the line shunt capacitances [5]. Such assumptions may introduce considerable error especially in the case of long lines. To provide more accurate results, an improved two-terminal fault location method is proposed in this part of the paper.

Let $\boldsymbol{z}$ and $\boldsymbol{y}$ denote the $3 \times 3$ per-unit length, series impedance and shunt admittance matrices of transmission line $i-j$. The equivalent $\pi$ model of this line can be obtained using the derivations provided in the literature (e.g., [40]). Let the series impedance and shunt admittance matrices of that line be denoted by $\boldsymbol{z}^{\prime}$ and $\boldsymbol{y}^{\prime}$. Given the line length $L, \boldsymbol{z}^{\prime}$ and $\boldsymbol{y}^{\prime}$ could

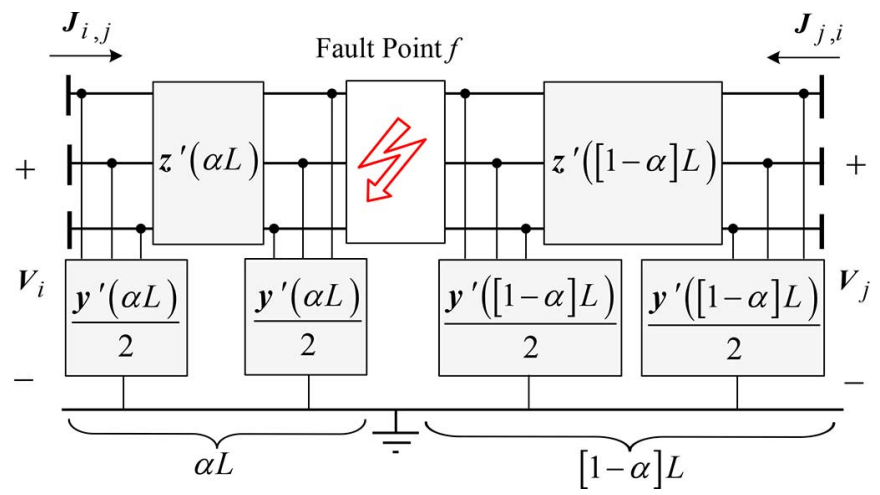

Fig. 1. General post-fault circuit of a multi-conductor transmission line represented by equivalent $\pi$ model.

be linearly approximated by $L \times \boldsymbol{z}$ and $L \times \boldsymbol{y}$. Although this approximation may work well for short and medium-length lines, a more accurate approximation is needed for long lines [40]. To accomplish this, the Taylor series expansion of the distributed-model line parameters should be used.

Let $3 \times 1$ column vectors $\boldsymbol{V}_{i}$ and $\boldsymbol{J}_{i, j}$ respectively denote the three-phase voltages at bus $i$, and sending-end currents of line $i$ - $j$. From the transmission line theory, the known link between the line two ports can be derived as

$$
\underbrace{\left[\begin{array}{c}
\boldsymbol{V}_{i} \\
\boldsymbol{J}_{i, j}
\end{array}\right]}_{6 \times 1}=\underbrace{\left[\begin{array}{cc}
\boldsymbol{I}+\frac{\boldsymbol{z}^{\prime} \boldsymbol{y}^{\prime}}{2} & \boldsymbol{z}^{\prime} \\
\boldsymbol{y}^{\prime}\left[\boldsymbol{I}+\frac{\boldsymbol{z}^{\prime} \boldsymbol{y}^{\prime}}{4}\right] & \boldsymbol{I}+\frac{\boldsymbol{y}^{\prime} \boldsymbol{z}^{\prime}}{2}
\end{array}\right]}_{6 \times 6} \underbrace{\left[\begin{array}{c}
\boldsymbol{V}_{j} \\
-\boldsymbol{J}_{j, i}
\end{array}\right]}_{6 \times 1}
$$

The first matrix on the right hand side of (1) is the transmission matrix of a length $L$ of line $i-j$ and is denoted by $\boldsymbol{T}_{i, j}(L)$, hereinafter.

By defining $\boldsymbol{A}=\boldsymbol{B}^{2}=\boldsymbol{z} \boldsymbol{y}$ and $\boldsymbol{Z}^{c}=\boldsymbol{B}^{-1} \boldsymbol{z}$, it is possible to write elements of $\boldsymbol{T}_{i, j}(L)$ in terms of hyperbolic functions as

$$
\begin{aligned}
\boldsymbol{T}_{i, j}(L) & =\left[\begin{array}{cc}
\boldsymbol{T}_{i, j}^{1}(L) & \boldsymbol{T}_{i, j}^{2}(L) \\
\boldsymbol{T}_{i, j}^{3}(L) & \boldsymbol{T}_{i, j}^{4}(L)
\end{array}\right] \\
& =\underbrace{\left[\begin{array}{cc}
\cosh (\boldsymbol{B} L) & \sinh (\boldsymbol{B} L) \boldsymbol{Z}^{c} \\
{\left[\boldsymbol{Z}^{c}\right]^{-1} \sinh (\boldsymbol{B} L)} & {\left[\boldsymbol{Z}^{c}\right]^{-1} \cosh (\boldsymbol{B} L) \boldsymbol{Z}^{c}}
\end{array}\right]}_{6 \times 6}
\end{aligned}
$$

The Taylor series expansions of the above sub-matrices are provided in Appendix $A$.

Let consider the circuit shown in Fig. 1. It illustrates a shortcircuit fault at point $f$ on line $i-j$. The transmission matrices of the two sections of this line with lengths $\alpha L$ and $[1-\alpha] L$ can be easily calculated using (2). Regardless of the fault type and its current, voltage at the fault point can be obtained by applying KVL from either end of the line. From (1) and (2), the voltage phasors at the fault point can be derived as

$$
\begin{aligned}
\boldsymbol{V}_{f}=\boldsymbol{T}_{i, j}^{1} & (\alpha L) \boldsymbol{V}_{i}-\boldsymbol{T}_{i, j}^{2}(\alpha L) \boldsymbol{J}_{i, j} \\
& =\boldsymbol{T}_{i, j}^{1}([1-\alpha] L) \boldsymbol{V}_{j}-\boldsymbol{T}_{i, j}^{2}([1-\alpha] L) \boldsymbol{J}_{j, i}
\end{aligned}
$$

The above equation can be expanded as shown in (4) at the bottom of the next page, where $\boldsymbol{a}_{n}, \boldsymbol{b}_{n}, \boldsymbol{c}_{n}$ and $\boldsymbol{d}_{n}$ are $3 \times 1$ vectors whose elements are provided in Appendix $B$.

Both sides of (4) have infinite number of terms. However, the higher the degree of $\alpha$, the lower the magnitude of its coefficient. 
The reason is the factorial terms in the denominators. Equation (4) is expanded only to the fourth degree of $\alpha$, in this study. Simulation results show that further developments of (4) do not result in significant improvement of the fault location accuracy. As will be shown later, the estimated fault location for long lines would remain quite accurate despite truncating the higher order terms. Accordingly, a system of three polynomial equations of order four, is obtained for the unknown variable $\alpha$ as below

$$
\underbrace{\left[\begin{array}{lllll}
\boldsymbol{h}_{0} & \boldsymbol{h}_{1} & \boldsymbol{h}_{2} & \boldsymbol{h}_{3} & \boldsymbol{h}_{4}
\end{array}\right]}_{3 \times 5} \times\left[\begin{array}{ccccc}
1 & \alpha & \alpha^{2} & \alpha^{3} & \alpha^{4}
\end{array}\right]^{T}=0,
$$

where the derivations of $\boldsymbol{h}_{0}$ to $\boldsymbol{h}_{4}$ are provided in Appendix $C$.

\section{B. Extension to Meshed Transmission Networks}

As proposed by Fortescue, the fault analysis of a three-phase power system could be much simpler in sequence domain, if the matrices of internal impedances of all system components are circular [41]. In this respect, the bus impedance matrices of sequence circuits can be used to obtain the current and voltage signals at different locations. Nonetheless, the sequence domain analysis of untransposed transmission networks is not of great help since the resulting sequence circuits would remain coupled. On the other hand, the pre-fault system unbalances of such networks could result in considerable amount of error, if neglected.

For untransposed networks, hence, the bus impedance matrix should be constructed in the phase-coordinates. The unknowns of the problem (fault location and impedances) have to be considered in constructing the bus impedance matrix. The elements of this matrix, and thus the resulting circuit equations, are highly nonlinear in terms of these unknowns. The technique firstly proposed in [31] is generalized here to make the pre-fault impedance matrix applicable even for the faulted network.

Let denote the pre-fault and post-fault variables by the superscripts "pre" and "post", respectively. Considering a linear behavior of the grid before and after the fault, the nodal equations for the untransposed three-phase power network prior to the fault can be written in the matrix form as

$$
\underbrace{V^{p r e}}_{3 N \times 1}=\underbrace{Z^{p r e}}_{3 N \times 3 N} \underbrace{I^{p r e}}_{3 N \times 1}
$$

where $\boldsymbol{V}^{\text {pre }}$ is the vector of pre-fault bus voltage phasors and $\boldsymbol{Z}^{\text {pre }}$ is the bus impedance matrix in the normal operating condition. Besides, $\boldsymbol{I}^{\text {pre }}=\left[I_{1}^{a}, I_{1}^{b}, I_{1}^{c} \ldots, I_{N}^{a}, I_{N}^{b}, I_{N}^{c}\right]^{T}$ is the vector of nodal injected currents, and $N$ is the number of system buses.

As shown in Figs. 2 and 3, line $i-j$ in the post-fault condition can be replaced with six equivalent current sources injecting the same amounts of currents as the replaced line. This is possible in virtue of the substitution theorem. Let the superscript $(i, j)$ denote the bus impedance matrix and vector of nodal injected

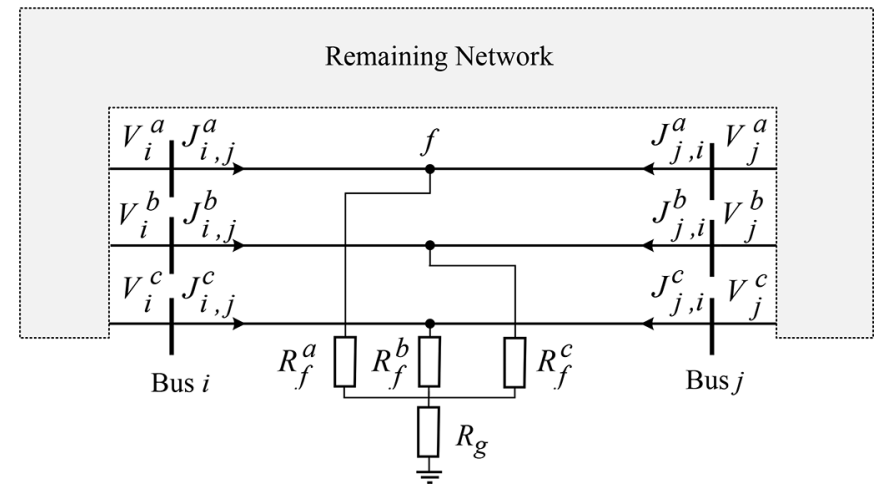

Fig. 2. General short-circuit fault on line $i-j$.

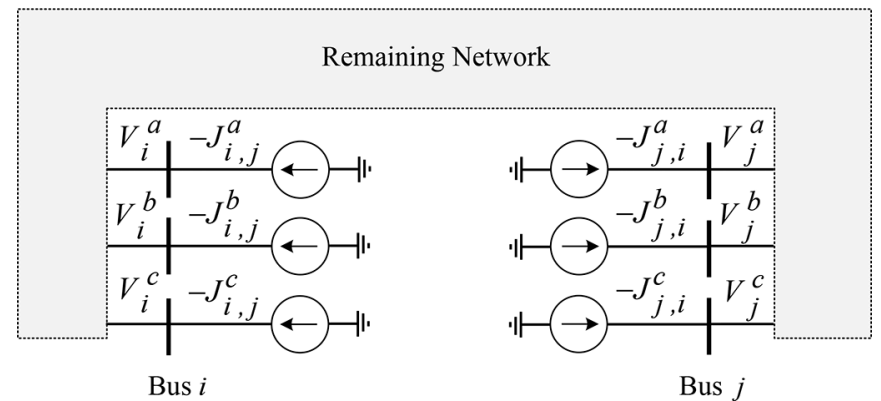

Fig. 3. Replacement of the faulted line $i-j$ with six suitably adjusted current sources.

currents after the faulted line is replaced with current sources. Therefore,

$$
\begin{aligned}
& \underbrace{V^{p r e}}_{3 N \times 1}=\underbrace{\boldsymbol{Z}^{(i, j)}}_{3 N \times 3 N} \underbrace{\boldsymbol{I}^{(i, j) p r e}}_{3 N \times 1}, \\
& \underbrace{\boldsymbol{V}^{\text {post }}}_{3 N \times 1}=\underbrace{\boldsymbol{Z}^{(i, j)}}_{3 N \times 3 N} \underbrace{\boldsymbol{I}^{(i, j) p o s t}}_{3 N \times 1} .
\end{aligned}
$$

Assume buses 1 to $n$ are equipped with PMUs. The superscript "meas" is used to denote synchrophasors obtained from PMUs. Subtracting pre-fault voltages from their post-fault values at a PMU-equipped bus, say bus $k$, yields

$$
\underbrace{\Delta \boldsymbol{V}_{k}^{\text {meas }}}_{3 \times 1}=\underbrace{\left[\boldsymbol{R}^{k(i, j)}\right]}_{3 \times 6} \underbrace{\left[\begin{array}{c}
\Delta \boldsymbol{J}_{i, j} \\
\Delta \boldsymbol{J}_{j, i}
\end{array}\right]}_{6 \times 1},
$$

where the $3 \times 1$ vectors $\Delta \boldsymbol{V}_{k}^{\text {meas }}, \Delta \boldsymbol{J}_{i, j}$, and $\Delta \boldsymbol{J}_{j, i}$ are expanded at the bottom of the next page.

If $R_{p, q}^{k(i, j)}$ denotes the entry in the $p$ th row and $q$ th column of matrix $\boldsymbol{R}^{k(i, j)}$, it is obtained from

$$
R_{p, q}^{k(i, j)}= \begin{cases}-Z_{3 k+p-3,3 i+q-3}^{(i, j)}, & 1 \leq q \leq 3 \\ -Z_{3 k+p-3,3 j+q-6}^{(i, j)}, & 4 \leq q \leq 6\end{cases}
$$

where the integer number $p$ varies from 1 to 3 .

$$
\sum_{n=0}^{\infty} \boldsymbol{a}_{n} \alpha^{2 n}+\sum_{n=0}^{\infty} \boldsymbol{b}_{n}[1-\alpha]^{2 n}=\sum_{n=0}^{\infty} \boldsymbol{c}_{n} \alpha^{2 n+1}+\sum_{n=0}^{\infty} \boldsymbol{d}_{n}[1-\alpha]^{2 n+1}
$$


If the voltage phasors of both ends of a non-faulted line is known, the equivalent $\pi$ model of that line can be used to determine its sending- and receiving-end current phasors. Let $\boldsymbol{J}_{(k)}$ be the vector of sending-end three-phase currents of line $u-w$. Given $\boldsymbol{Z}_{u, w}^{c}$ is the line characteristic impedance, the superimposed sending-end current of this line can be expressed as

$$
\begin{aligned}
& \underbrace{\Delta \boldsymbol{J}_{(k)}^{\text {meas }}}_{3 \times 1}=\left[\boldsymbol{Z}_{u, w}^{c}\right]^{-1} \operatorname{coth}\left(\boldsymbol{B}_{u, w} L_{u, w}\right) \Delta \boldsymbol{V}_{u} \\
& -\left[\sinh \left(\boldsymbol{B}_{u, w} L_{u, w}\right) \boldsymbol{Z}_{u, w}^{c}\right]^{-1} \Delta \boldsymbol{V}_{w}=\underbrace{\left[\boldsymbol{Q}^{k(i, j)}\right]}_{3 \times 6} \underbrace{\left[\begin{array}{c}
\Delta \boldsymbol{J}_{i, j} \\
\Delta \boldsymbol{J}_{j, i}
\end{array}\right]}_{6 \times 1},
\end{aligned}
$$

where $\boldsymbol{Q}^{k(i, j)}$ is obtained from

$$
\begin{aligned}
{\left[\boldsymbol{Q}^{k(i, j)}\right]=\left[\boldsymbol{Z}_{u, w}^{c}\right]^{-1} \operatorname{coth}\left(\boldsymbol{B}_{u, w} L_{u, w}\right) \boldsymbol{R}^{u(i, j)} } & \\
& -\left[\sinh \left(\boldsymbol{B}_{u, w} L_{u, w}\right) \boldsymbol{Z}_{u, w}^{c}\right]^{-1} \boldsymbol{R}^{w(i, j)}
\end{aligned}
$$

It should be noted that (11) is written with the assumption that line $u-w$ is not faulted. Expressing the faulted line terminal currents as a function of its terminal voltages is unfeasible unless the fault type, location and its impedance are known. Nevertheless, the flowing current from each side can be directly measured if the corresponding bus has been equipped with a PMU, as well. In case bus $i$ is equipped with a PMU, the faulted line sending-end current can be written as

$$
\Delta \boldsymbol{J}_{i, j}^{\text {meas }}=\Delta \boldsymbol{J}_{i, j}+\boldsymbol{e}_{i, j}
$$

where $\boldsymbol{e}_{i, j}$ denotes the error between the measured and true values of the sending-end current of line $i-j$. A similar equation could be also derived for the receiving-end current of that line, in case bus $j$ has been equipped with a PMU.

Let $l$ be the number of transmission line ends whose current phasors are measured by PMUs. Additionally, $l_{h}$ is considered to be the number of non-faulted line ends whose current phasors are measured by PMUs. Let $N_{s}$ denote the number of synchrophasor measurements provided by PMUs, i.e., $n+l$. Considering all constructible equations in any form of $(9),(11)$ and (13), a system of $N_{s}$ linear equations for six unknowns can be developed as

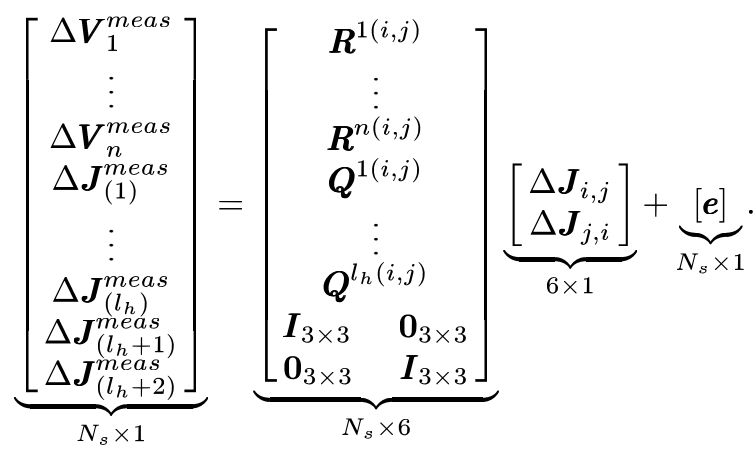

If any of the faulted line ends is not equipped with a PMU to measure that line currents, the corresponding equations should be excluded from the last six equations of (14).

It should be emphasized that the fault equations in (14) are not just limited to the synchrophasor measurements at the faulted line terminals. Conversely, the equations associated with measurements farther from the fault point are also included in this system. By doing so, the adverse effect of erroneous instrument transformers at the faulted line terminals on the fault location accuracy would be decreased. In virtue of the redundancy provided by the proposed formulation, bad data detection can also be deployed to achieve more accuracy. Accordingly, the erroneous fault equations would be readily identified and removed from the equations set of (14).

This system of equations would have a matrix form of

$$
\boldsymbol{M}=\boldsymbol{H} X+\boldsymbol{e},
$$

where $\boldsymbol{M}$ and $\boldsymbol{H}$ are the measurement and coefficient matrices, respectively. Besides, $\boldsymbol{X}$ is the matrix of unknowns that represents the six current sources substituted for the faulted line.

After calculation of $\Delta \boldsymbol{J}_{i, j}$ and $\Delta \boldsymbol{J}_{j, i}$ from (15), the threephase voltage synchrophasors at buses $i$ and $j$ can be calculated using (9) as

$$
\underbrace{\left[\begin{array}{c}
\Delta \hat{\boldsymbol{V}}_{i} \\
\Delta \hat{\boldsymbol{V}}_{j}
\end{array}\right]}_{6 \times 1}=\underbrace{\left[\begin{array}{c}
\boldsymbol{R}^{i(i, j)} \\
\boldsymbol{R}^{j(i, j)}
\end{array}\right]}_{6 \times 6} \underbrace{\left[\begin{array}{c}
\Delta \hat{\boldsymbol{J}}_{i, j} \\
\Delta \hat{\boldsymbol{J}}_{j, i}
\end{array}\right]}_{6 \times 1} .
$$

Now, the three-phase currents and voltages at both ends of the faulted line have been obtained from (15) and (16). Therefore, it is possible to readily locate the fault distance on the line using the improved two-terminal method described in the previous subsection.

Over-determined systems of equations have, in general, no solution. However, the superimposed current synchrophasors at the faulted line terminals is a solution for (15). This system can be solved using the linear least-squares method to obtain the values of the best six fitting current sources for each suspected line, denoted by $\hat{\boldsymbol{X}}$. The hat symbol on $\boldsymbol{X}$ implies that the estimated unknowns are not exactly the same as true unknowns $\boldsymbol{X}$ due to existence of the error vector $\boldsymbol{e}$ on the right hand side of (15). Nevertheless, such a solution ensures that the sum of squared residuals is minimal, where a residual is defined as the difference between the measurement in $\boldsymbol{M}$ and its corrected value obtained from $\boldsymbol{H} \hat{\boldsymbol{X}}$. The sum of squared residuals corresponding to the faulted line $i-j$ is obtained from

$$
E_{i, j}=[\boldsymbol{M}-\boldsymbol{H} \hat{\boldsymbol{X}}]^{T}[\boldsymbol{M}-\boldsymbol{H} \hat{\boldsymbol{X}}] .
$$

It should be pointed out that the current sources substituted for the actual faulted line are the unknowns for which (15) holds true. It means that $E_{i, j}$ is expected to take a negligible value for the faulted line, and large values for the other ones. Thus, the faulted line can be specified by comparing the calculated $E_{i, j}$ 's for examined transmission lines, since the smallest one corresponds to it.

$$
\Delta \boldsymbol{V}_{k}^{\text {meas }}=\left[\begin{array}{c}
\Delta V_{k}^{a} \\
\Delta V_{k}^{b} \\
\Delta V_{k}^{c}
\end{array}\right], \Delta \boldsymbol{J}_{i, j}=\left[\begin{array}{c}
\Delta J_{i, j}^{a} \\
\Delta J_{i, j}^{b} \\
\Delta J_{i, j}^{c}
\end{array}\right] \text { and } \Delta \boldsymbol{J}_{j, i}=\left[\begin{array}{c}
\Delta J_{j, i}^{a} \\
\Delta J_{j, i}^{b} \\
\Delta J_{j, i}^{c}
\end{array}\right]
$$




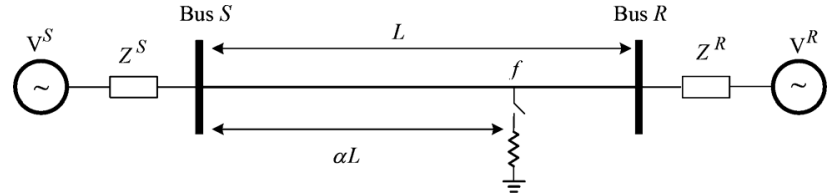

Fig. 4. Single-line diagram of the untransposed two-terminal transmission system.

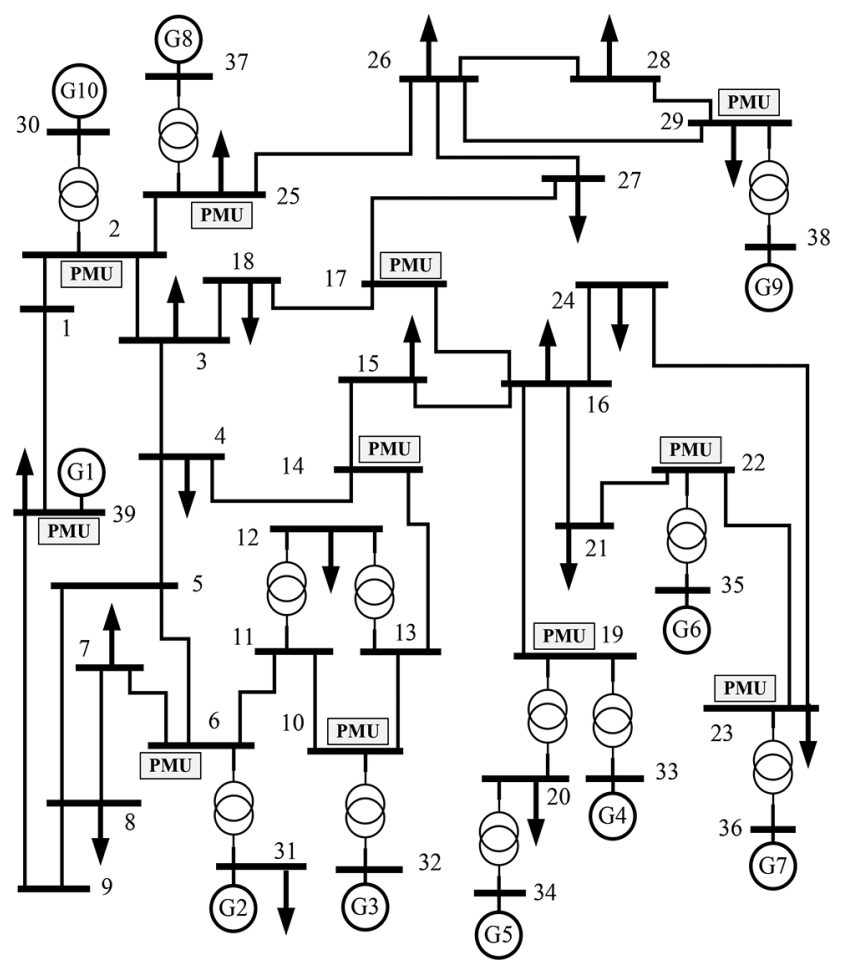

Fig. 5. Single-line diagram of the untransposed 39-bus test system.

The superimposed current and voltage at the faulted line ends, along with the faulted sections transmission matrices $\boldsymbol{T}(\alpha L)$ and $\boldsymbol{T}((1-\alpha) L)$ can be used to obtain the fault path current on that line. Let $J_{f, i}^{a}, J_{f, i}^{b}$ and $J_{f, i}^{c}$ denote the faulted line currents at the fault point, estimated from the bus $i$ side. Similarly are defined $J_{f, j}^{a}, J_{f, j}^{b}$ and $J_{f, j}^{c}$. A phase is inferred to be faulted, if the sum of its two side currents at the fault point is non-zero.

\section{Performance Evaluation}

The performance of the proposed algorithm has been assessed via offline simulations. In particular, this section illustrates separately the performance assessment of proposed two-terminal fault location and its extension to wide-area process. The untransposed two-terminal transmission system, and the untransposed New England 39-bus system [42] are selected as test systems. The single-line diagrams of these two systems are shown in Figs. 4 and 5, respectively. The parameters of the transmission lines used to construct these test systems are provided in Appendix $D$. The two-terminal transmission system is used to specifically compare the proposed two-terminal method with the conventional transposed and untransposed methods, and justify its superiority over those methods. Afterward, the 39-bus test system has been used to demonstrate the effectiveness of the proposed wide-area fault location algorithm.
Appropriate software is used to model the two-terminal [43], and 39-bus [44] test systems operating at nominal frequency of $50 \mathrm{~Hz}$. To imitate the synchrophasor extraction process, the generated signals are passed through an anti-aliasing Butterworth filter with cutoff frequency of $400 \mathrm{~Hz}$ and then, sampled with a sampling frequency of $2500 \mathrm{~Hz}$. In order to extract the fundamental-frequency component of signals, the discrete Fourier transform (DFT) along with a digital mimic filter is used. Next, the obtained signals are averaged from 1.5 to 3 cycles after the fault inception. As a commonly used metric to show the fault location accuracy, the relative estimation error is finally calculated as follows [1]:

$$
\text { Error }(\%)=\frac{\mid \text { Actual location-Estimated location } \mid}{\text { Faulted line lenght }} \times 100 \text {. }
$$

All calculations have been carried out in the MATLAB environment on a $2.4 \mathrm{GHz}$ dual-core processor with $4 \mathrm{~GB}$ of RAM. A single complex multiplication takes around $T_{m}=5 \mu \mathrm{s}$ in this platform. For the improved two-terminal fault location algorithm proposed in this paper, the construction and subsequent solution of (5) take around $T_{p}=25 \mathrm{~ms}$ using the trust-region algorithm called by "fsolve" MATLAB function. The 11 PMUs installed in the 39-bus test system provides 135 synchrophasor measurements (three-phase current and voltage phasors) and all 34 lines are examined to identify the faulted line. The total computational time for each fault case is about 1.05 second on average, which agrees with the 1.15 second upper boundary obtained from the theoretical formula given in Appendix $E$.

\section{A. Proposed Two-Terminal Fault Location Method}

The key point in the proposed two-terminal method is to use a sufficiently accurate model of unbalanced transmission line parameters. In this part, the performance of this method is compared with that of the conventional transposed [4] and untransposed [1], [5], [45]-[47] fault location methods. To apply the conventional transposed method to untransposed lines, the mutual couplings between sequence circuits are ignored. Thus, three distinct estimations are obtained for the fault location corresponding to the zero, positive and negative sequence circuits. These estimations are not, in general, real numbers but complex. The estimation demonstrated for the conventional transposed method is obtained by averaging the absolute value of estimations in the three sequence networks.

Contrary to the conventional transposed method which assumes the line is ideally transposed, the conventional untransposed method takes into account the line being untransposed. In this method, series impedances of the two sections of faulted line are set equal to their linear approximations. For applying the conventional untransposed method, the linear approximation of the line shunt admittances are also taken into account in this section. However, as explained earlier, the proposed method uses a more accurate approach which results in the solution of a system of three quartic equations.

The untransposed two-terminal transmission system shown in Fig. 4 is used to evaluate the proposed and the conventional transposed [4] and untransposed [5] two-terminal fault location methods. The associated data and parameters of this $345 \mathrm{kV}$ system are provided in Appendix $F$. By setting the line length 


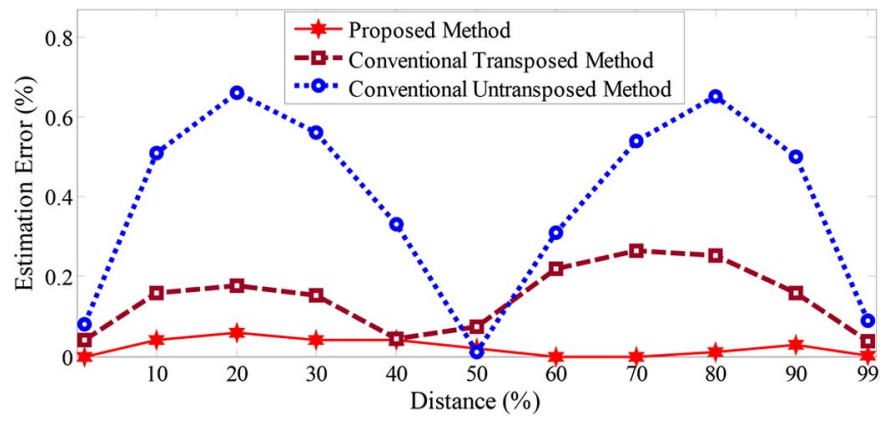

Fig. 6. Fault location on a $400 \mathrm{~km}$ untransposed transmission line using the proposed, conventional transposed and untransposed methods.

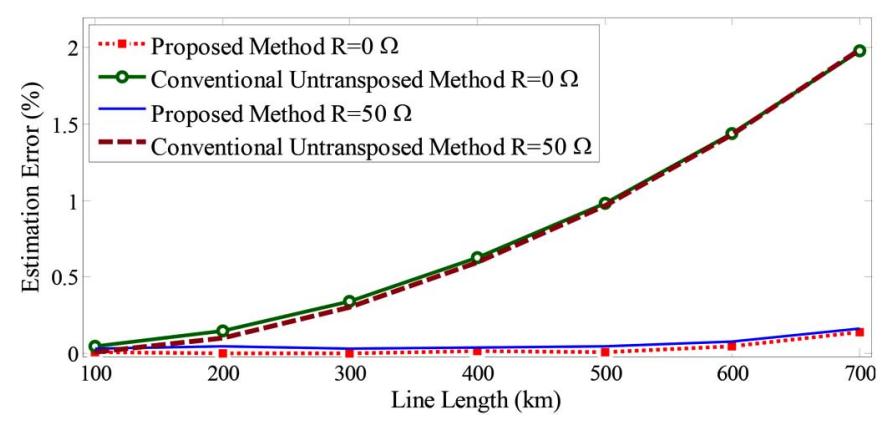

Fig. 7. Estimation error comparison between the proposed and conventional untransposed methods, for a 1-ph-g fault at $75 \%$ of lines with different lengths.

equal to $400 \mathrm{~km}$, a number of 1-ph-g faults are applied at eleven locations along this line. Fig. 6 illustrates the estimation errors resulting from the various methods. The proposed method provides the most accurate results compared to the other ones. It can be also concluded that the conventional untransposed method might even give less accurate result than that of methods basically developed for transposed lines such as [4].

To better compare the performance of the proposed method and conventional untransposed method in fault location on untransposed transmission lines, a number of 1-ph-g and 2-ph fault cases are simulated on $75 \%$ of several lines with various lengths. Although the fault resistance in transmission levels mostly lies between 0 to $10 \Omega$, it might exceed this range in some cases [1]. Therefore, larger values for fault resistance are commonly examined in the literature to verify the algorithm robustness against this important parameter. In this respect, two fault resistances of 0 and $50 \Omega$ are examined. Figs. 7 and 8 show the relative estimation error for different line lengths from 100 to $700 \mathrm{~km}$. As shown, the proposed method provides more accurate results especially for long lines.

Amongst many fault location methods that have been proposed so far, few are capable to deal with untransposed lines. In fact, existing fault location methods for untransposed lines have been developed based on the same simplifications that have been made in [5]. Recent works have focused on other aspects of the problem such as synchronization of measured phasors [46] or line parameters errors [47], and/or have extended the conventional method to deal with double-circuit and series-compensated untransposed lines. In this respect, a comparison between the fault location results obtained by the proposed method and approaches of these research works is performed. For this analysis, faults are simulated at four locations on transmission lines

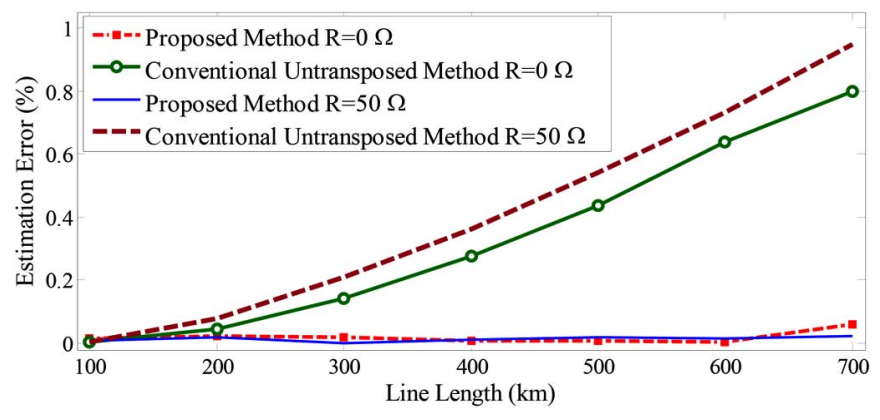

Fig. 8. Estimation error comparison between the proposed and conventional untransposed methods, for a 2 -ph fault at $75 \%$ of lines with different lengths.

TABLE I

Average Estimation ERROR FOR 1-PH-G FAULTS With A $10 \Omega$ Fault Resistance Using Different Methods

\begin{tabular}{c||c|c|c}
\hline \multicolumn{1}{c||}{ Line Length $(\mathrm{km})$} & 150 & 300 & 450 \\
\hline \hline \multicolumn{1}{c||}{ Method } & \multicolumn{3}{|c}{ Average Estimation Error \% } \\
\hline Proposed & 0.02 & 0.03 & 0.06 \\
\hline$[1]$ and [45] & 0.09 & 0.28 & 0.57 \\
\hline$[46]$ & 0.10 & 0.30 & 0.64 \\
\hline$[47]$ & 0.08 & 0.17 & 0.52 \\
\hline
\end{tabular}

with 150, 300 and $450 \mathrm{~km}$ lengths. Besides, the fault resistance is assumed to be $10 \Omega$. The obtained results are summarized in Table I.

It can be confirmed that the proposed two-terminal method over-performs the other approaches for all the considered line lengths. The reason for this superiority is the use of more accurate approximations of the equivalent $\pi$ model of the faulted line. Deficiency of the linear approximation becomes more noticeable in the case of long lines, especially for lines with high voltage levels and with large shunt capacitances.

\section{B. General Evaluation of the Proposed Wide-Area Algorithm}

In this part, the proposed wide-area fault location algorithm performance is evaluated with respect to different factors. The untransposed 39-bus system shown in Fig. 5 has been chosen as the test system.

1) Deficiency of Impedance-Based Wide-Area Methods: Amongst the existing wide-area fault location methods, some enforce a set of constraints on the PMU installation pattern and, in general, use one or two of closest measurements to the fault point for fault location [20]-[22]. Therefore, their performance is highly affected by transient response of instrument transformers similar to conventional methods. Besides, losing one or more PMU would make these methods not applicable. A series of other methods [23]-[26] compare the waveforms recorded during the fault with a simulation database built offline. Considering all the possible fault locations, resistances and fault types, it is not feasible to create such a huge database for relatively large-scale power systems. For some other methods [27] and [28], not more than two measurements can be used at the same time, unless a nonlinear optimization problem is solved [29]. A major disadvantage of the mentioned wide-area fault location methods is that bad data detection techniques cannot be readily incorporated in them. Reference [30] pinpoints the 
TABLE II

FAUlt Location RESUlts With AND Without CONSIDERING TRANSMISSION LINES UNBALANCES

\begin{tabular}{c||c|c|c|c}
\hline \multicolumn{1}{c||}{ Fault Location Method } & \multicolumn{2}{c|}{ Proposed } & \multicolumn{2}{c}{ Reference [31] } \\
\hline \hline \multirow{2}{*}{ Line Untransposition } & \multicolumn{3}{c}{ Considered } & \multicolumn{2}{c}{ Not Considered } \\
\hline \hline \multirow{2}{*}{ Fault Type } & \multicolumn{3}{c}{ Estimation Error \% } \\
\cline { 2 - 5 } & Ave. & Max. & Ave. & Max. \\
\hline 1-ph-g & 0.29 & 0.71 & 3.01 & 6.13 \\
\hline 2 -ph & 0.24 & 0.58 & 2.28 & 5.22 \\
\hline 2 -ph-g & 0.25 & 0.65 & 2.45 & 5.68 \\
\hline 3-ph-g & 0.21 & 0.52 & 1.81 & 4.86 \\
\hline All in Total & 0.25 & --- & 2.36 & --- \\
\hline
\end{tabular}

TABLE III

Fault Location Results in the Fully ObSERVABle Network

\begin{tabular}{c||c|c|c}
\hline Fault Resistance $(\Omega)$ & 0 & 25 & 50 \\
\hline \hline Fault Type & \multicolumn{3}{|c}{ Average Estimation Error \% } \\
\hline 1-ph-g & 0.24 & 0.25 & 0.27 \\
\hline 2-ph & 0.20 & 0.20 & 0.22 \\
\hline 2-ph-g & 0.21 & 0.23 & 0.24 \\
\hline 3-ph-g & 0.15 & 0.18 & 0.19 \\
\hline All in Total & 0.20 & 0.21 & 0.23 \\
\hline \multicolumn{4}{|l}{}
\end{tabular}

fault location using a trial-and-error process by examining a huge number of possible fault distances and resistances.

The more recent and effective fault location methods [31] and [32], use the available PMU data and give rise to almost the same accurate results. Furthermore, bad data detection can be readily deployed in these methods to achieve the highest possible accuracy. Extensive simulations conducted by the authors show that even such effective methods cannot properly deal with faults on untransposed transmission networks. The reason is quite trivial since these methods are inherently designed for transposed transmission lines. Due to space limit, only the obtained results by [31] for faults on line 26-28 has been reported and discussed in this part. It should be noted that almost the same inexact results have been obtained using the method proposed in [32].

Table II summarizes the average and maximum estimation errors of more than 800 fault cases. Different fault types have been applied at various points on line $26-28$ by moving the fault location along that line with steps of $0.05 \mathrm{pu}$. The effectiveness of the proposed algorithm can be easily confirmed by comparing the second and fourth columns of Table II. It can be observed that the average estimation error of the proposed algorithm is about $0.25 \%$. Nonetheless, neglecting the line unbalances results in inexact approximation of the current sources replaced for the faulted line. Consequently, the voltage at the faulted line ends could not be accurately obtained. In some cases, the estimated fault location would have up to $6 \%$ difference with its actual value. In fact, the proposed method provides highly accurate results since it rigorously takes into account the untransposed line parameters to formulate the problem.

2) Fault Location on the Fully Observable Network: As shown in Fig. 5, the 39-bus system is equipped with 11 PMUs making it fully observable [48], [49]. To scrutinize the proposed fault location algorithm, a total of more than 2500 short-circuit cases have been conducted on this system. To make the evaluation comprehensive, various fault types with different fault
TABLE IV

Three-Phase Voltage and Current Signals at Terminals of the Faulted Line 21-22 Calculated Based on PMU Data

\begin{tabular}{|c|c|c|}
\hline Synchrophasor & True Values & Estimated Values \\
\hline$\Delta \boldsymbol{J}_{21-22}(\mathrm{kA})$ & {$\left[\begin{array}{c}0.661-10.262 \mathrm{i} \\
0.064-0.274 \mathrm{i} \\
0.017-0.040 \mathrm{i}\end{array}\right]$} & {$\left[\begin{array}{c}0.662-10.261 \mathrm{i} \\
0.065-0.273 \mathrm{i} \\
0.017-0.041 \mathrm{i}\end{array}\right]$} \\
\hline$\Delta \boldsymbol{J}_{22-21}(\mathrm{kA})$ & {$\left[\begin{array}{c}1.098-12.553 \mathrm{i} \\
-0.065+0.270 \mathrm{i} \\
-0.0181+0.036 \mathrm{i}\end{array}\right]$} & {$\left[\begin{array}{c}1.096-12.553 \mathrm{i} \\
-0.068+0.271 \mathrm{i} \\
-0.0182+0.036 \mathrm{i}\end{array}\right]$} \\
\hline$\Delta V_{21}(\mathrm{kV})$ & $\left.\begin{array}{l}-106.58+32.307 \mathrm{i} \\
-41.133+11.366 \mathrm{i} \\
-39.518+11.091 \mathrm{i}\end{array}\right]$ & $\begin{array}{c}-106.65+32.31 \mathrm{i} \\
-41.125+11.36 \mathrm{i} \\
-39.521+11.084 \mathrm{i}\end{array}$ \\
\hline$\Delta V_{22}(\mathrm{kV})$ & {$\left[\begin{array}{c}-36.257+28.121 \mathrm{i} \\
-11.560+2.834 \mathrm{i} \\
-12.066+3.249 \mathrm{i}\end{array}\right]$} & {$\left[\begin{array}{c}-36.261+28.13 \mathrm{i} \\
-11.562+2.84 \mathrm{i} \\
-12.082+3.245 \mathrm{i}\end{array}\right]$} \\
\hline
\end{tabular}

resistances from 0 to $50 \Omega$ are simulated at five points on each transmission line. Table III summarizes the obtained results for the simulated cases. As shown, the average estimations error is less than $0.27 \%$ in all cases. Even for large fault resistances, the estimation accuracy is quite acceptable. The reason is that the proposed algorithm solves the fault equations by omitting the fault resistance. This has been realized by using synchrophasors collected from different locations of the system.

In rare cases, the proposed method misdiagnoses a neighbor line as faulted instead of the actual faulted one. In more than 2500 simulations conducted in this part, such a problem has happened only in 20 close-in fault cases and in areas sparsely covered with PMUs. In these cases, the estimated fault location is at the beginning of a neighbor line, which is physically very close to the actual fault point. It should be noted that the one- or two-terminal methods are not effective for such close-in faults, either. Nonetheless, simulation results confirm that the proposed method could accurately locate these close-in faults if the faulted line is known.

One of the case studies has been selected to provide a stepby-step explanation on how to apply the proposed algorithm to identify the faulted line and pinpoint the fault distance on it. In this case, a 1-ph-g fault occurs at $40 \%$ of line $21-22$ of the system. First, the system of (15) is solved for every 34 line using $\boldsymbol{X}=\left[\boldsymbol{H}^{\mathrm{T}} \boldsymbol{H}\right]^{-1} \boldsymbol{H}^{\mathrm{T}} \boldsymbol{M}$. This gives an estimate for three-phase current at two ends of the substituted transmission line. The true and estimated current phasors corresponding to the faulted line 21-22 have been tabulated in the first two rows of Table IV. These values can be used in (16) to obtain three-phase voltages of faulted line terminals as given in the last two rows of Table IV.

In the next step, the system of (5) is constructed and solved for every line based on the voltage and current synchrophasors calculated for that line. Amongst all transmission lines, only the calculated fault distance for five lines is real and lies in the valid range of $[0,1]$. For the remaining lines, the calculated fault distance is either complex, or its real part is negative or larger than 1 . The sum of squared residuals (17) is calculated for the lines whose estimated fault distances lie within the acceptable 
TABLE V

FAULT LOCATION RESULTS ON LINE 4-14 CONSIDERING INSTRUMENT TRANSFORMERS TRANSIENT RESPONSE

\begin{tabular}{c||c|c|c|c|c|c|c|c}
\hline \multicolumn{1}{c||}{ Fault Distance (\%) } & 5 & 15 & 30 & 50 & 70 & 85 & 95 \\
\hline Fault Location Method & \multicolumn{7}{|c|}{ Fault Location Percentage Error (\%) } \\
\hline Conventional [5] & 3.71 & 2.58 & 0.63 & 0.24 & 0.48 & 1.47 & 2.69 \\
\hline Proposed Wide-Area & 0.30 & 0.26 & 0.21 & 0.21 & 0.25 & 0.26 & 0.27 \\
\hline
\end{tabular}

TABLE VI

Number of Reguired Measurement Devices for Being Able to Locate FAULTS ON EVERY LINE BY DifFERENT METHODS

\begin{tabular}{c||c|c|c}
\hline Fault Location Method & Proposed & One-Terminal & Two-Terminal \\
\hline $\begin{array}{c}\text { Number of Required } \\
\text { Measurement Devices }\end{array}$ & 11 & 34 & 68 \\
\hline
\end{tabular}

range. The least sum of squared residual, which is $2.4 \times 10^{-2}$, corresponds to the faulted line. The next least value amongst the calculated ones is 1.56 . Subsequently, line $21-22$ is identified as being faulted and the distance $40 \%$ on it is determined to be the fault location.

3) Transient Response of Instrument Transformers: One of the advantages of the proposed wide-area fault location method over the conventional methods is that it provides the possibility of identifying and removing erroneous measurements in virtue of containing several redundant fault equations. While the conventional methods become highly inaccurate in case of close-in faults [1], bad PMU data could be readily identified and removed in the linear least-squares framework of the proposed method, using the Normalized Residual bad data detection test [50]. Besides, PMUs that are located farther from the fault point would experience smaller changes due to the fault compared to measurement devices right at the faulted line terminals. This is important because the smaller the change an instrument transformer undergoes due to a fault, the shorter is its transient response and, thereby, the faster it would be in reproducing an exact replica of its primary input [51].

To investigate the influence of instrument transformers transient response on the fault location accuracy, different fault types have been examined on line 4-14. This line has been selected to be studied since it has a PMU at terminal 14 making it prone to transient response of instrument transformers in the case of close-in faults. Furthermore, the Normalized Residual test has been used for bad data detection. Table $\mathrm{V}$ provides the calculated average errors using the proposed and conventional untransposed fault location methods [5]. The obtained results confirm that only the proposed method could provide accurate results even with the inclusion of inexact measurements.

It should be also pointed out that the proposed method needs less number of measurement devices compared to conventional fault location methods. This holds in addition to its better performance in the case of transient response of instrument transformers. Table VI shows the number of measurement devices needed for being able to apply one-terminal, two-terminal and proposed fault location methods to all lines of the 39-bus test system.

4) Fault Location With Limited PMU Coverage: PMU placement can be the objective of several optimization problems such
TABLE VII

Fault Location Results in the PARTially OBSERVABLE Network

\begin{tabular}{c||c|c|c}
\hline \multicolumn{1}{c||}{ Fault Resistance $(\Omega)$} & 0 & 25 & 50 \\
\hline \hline \multicolumn{1}{c||}{ Fault Type } & \multicolumn{3}{|c}{ Average Estimation Error \% } \\
\hline 1-ph-g & 0.59 & 0.63 & 0.67 \\
\hline 2-ph & 0.44 & 0.46 & 0.50 \\
\hline 2-ph-g & 0.55 & 0.56 & 0.59 \\
\hline 3-ph-g & 0.32 & 0.38 & 0.41 \\
\hline All in Total & 0.48 & 0.51 & 0.54 \\
\hline
\end{tabular}

as network observability [49], state estimation accuracy [52], inter-area oscillation monitoring and damping and etc. Therefore, transmission line operators might not install PMUs only to maximize the performance of a wide-area fault location functionality. What we actually have assumed in this paper is to make use of the existing PMU set for wide-area fault location without enforcing any constraint on the PMU installation pattern. The network observability is not a necessary condition for wide-area fault location [31]. Theoretically, even six synchrophasor measurements suffice for (15) to be solvable, and hence, to locate the fault using the proposed algorithm. However, it is reasonable to expect more accurate results using a larger number of PMUs.

To study the fault location accuracy with limited PMU coverage, a new PMU set is considered at buses 2, 6, 14, 17, 19, 22 and 29 of the 39-bus test system. This set does not make the system fully observable. Nevertheless, the faulted line is successfully diagnosed in more than $96 \%$ of the simulated cases. It should be noted that this performance is still quite better than the performance of the existing wide-area fault location methods. The reason is that all of those methods are formulated with the assumption that the network transmission lines are completely transposed. Table VII shows the summary of fault location for the correctly diagnosed cases. As tabulated, the average error is less than $0.7 \%$, even for high impedance faults. It can be observed from the table that the estimation accuracy slightly decreases due to the limited PMU coverage, as expected. However, the results still remain more than acceptable from a practical point of view.

5) Robustness Against Synchrophasors Errors: To take into account the synchrophasor estimation errors, a maximum total vector error (TVE) of $1 \%$ has been considered based on the IEEE standard [53]. Accordingly, synchrophasor measurements must be synchronized with a maximum time error of $\pm 31 \mu \mathrm{s}$ for a $50 \mathrm{~Hz}$ system. The phasors amplitude and angle errors are considered to have a normal distribution with mean zero. Their corresponding standard deviations have also been set based on an error variation range of $\pm 1 \%$ and considering a confidence interval of $99.7 \%$.

Fig. 9 depicts the obtained results for a total of $100001-\mathrm{ph}-\mathrm{g}$ fault cases at $60 \%$ of line $15-16$. The resulting estimation error demonstrates a normal distribution with mean and standard deviation of $0.3 \%$ and $0.24 \%$, respectively. From a practical point of view, the obtained results are quiet acceptable.

6) Time-Varying Arc Resistance: To evaluate the proposed method performance in the case of time-varying arc resistance, a number of faults with such resistances are simulated in this part. To model the time-varying arc, its dynamic volt-ampere 


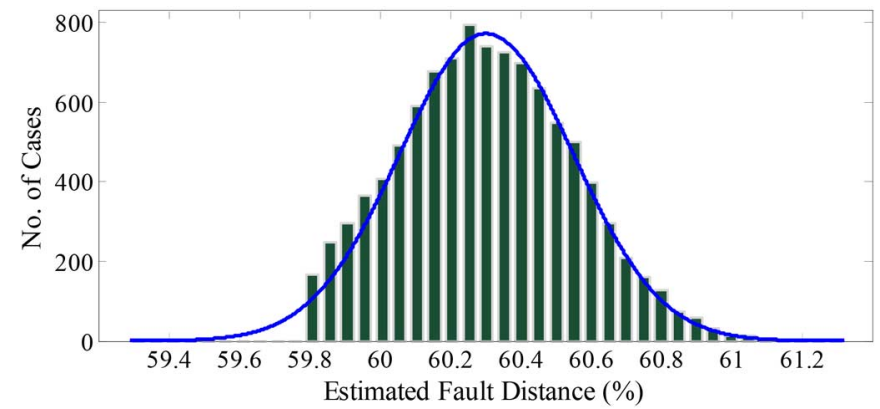

Fig. 9. Influence of synchrophasor errors on the fault location accuracy for a 1-ph-g fault at $60 \%$ of line $15-16$.

TABLE VIII

FAULT LOCATION RESUlTS FOR 1-PH-G FAULTS ON LINE 15-16 WITH FIXED RESISTANCE AND TIME-VARYING ARC

\begin{tabular}{c||c|c|c|c|c}
\hline \multicolumn{1}{c||}{ Fault Resistance } & \multicolumn{5}{c}{ Fault Distance \% } \\
\cline { 2 - 6 } & 10 & 30 & 50 & 70 & 90 \\
\hline $25 \Omega$ & 0.28 & 0.27 & 0.25 & 0.30 & 0.32 \\
\hline Time-Varying Arc & 0.75 & 0.69 & 0.54 & 0.73 & 1.12 \\
\hline
\end{tabular}

characteristic is taken into account based on the empirical differential equation described in [1]. Various distances on line 15-16 of the 39-bus test system are examined for 1-ph-g faults with this time-varying resistance, and also for a constant $25 \Omega$ resistance. The obtained results are summarized in Table VIII. As expected, the time-varying nature of the arc has slightly worsened the fault location accuracy. However, the obtained results are quite acceptable from a practical point of view.

\section{CONCLUSION}

A wide-area fault location algorithm has been proposed in this paper for untransposed transmission networks using voltage and current synchrophasors provided by PMUs. Despite being inherently nonlinear in terms of fault distance and impedance, the fault equations are made linear using six suitably-defined auxiliary variables. Accordingly, three-phase voltages and currents at the faulted line ends are obtained with no direct measurements. A two-terminal fault location method has been also proposed to take advantage of the calculated phasors for locating the fault more accurately. The proposed methodology there, i.e., the use of truncated Taylor series to model the equivalent $\pi$ circuit of the line, can be applied to other fault location problems related to untransposed lines. For example, fault location on double-circuit and series-compensated untransposed lines can be readily dealt with by such an approach in order to improve the estimation accuracy.

The proposed wide-area fault location algorithm is characterized by a low computational complexity. Such peculiarity might be an asset that allows, in principle, its coupling with real-time operation processes and its adoption as a back-up protection if a fast and reliable telecommunication infrastructure is available.

The major features of the proposed algorithm can be summarized as follows:

- The proposed algorithm is general and can be applied to transposed or untransposed networks in order to locate symmetrical/asymmetrical faults.

- The algorithm identifies the faulted line and does not require it as an input.

- A limited number of pre- and post-fault synchrophasors are sufficient to determine the fault location and type.

- Obtained fault location estimations are quite accurate even for long lines and/or high impedance faults.

- It is computationally effective and can be used for fast fault location.

- The proposed method is robust against transient response of instrument transformers and phasor estimation errors.

\section{APPENDIX}

A) : Elements of transmission matrix $\boldsymbol{T}_{i, j}(L)$ in (2) are obtained from:

$\boldsymbol{T}_{i, j}^{1}(L)=\sum_{n=0}^{\infty} \frac{\boldsymbol{A}^{n} L^{2 n}}{2 n !}, \quad \boldsymbol{T}_{i, j}^{2}(L)=\sum_{n=0}^{\infty} \frac{\boldsymbol{A}^{n} \boldsymbol{z} L^{2 n+1}}{(2 n+1) !}$
$\boldsymbol{T}_{i, j}^{3}(L)=\sum_{n=0}^{\infty} \frac{\boldsymbol{z}^{-1} \boldsymbol{A}^{n+1} L^{2 n+1}}{(2 n+1) !} \operatorname{and} \boldsymbol{T}_{i, j}^{4}(L)=\sum_{n=0}^{\infty} \frac{\boldsymbol{z}^{-1} \boldsymbol{A}^{n} \boldsymbol{z} L^{2 n}}{2 n !}$

B) : Coefficient vectors of $\boldsymbol{a}_{n}, \boldsymbol{b}_{n}, \boldsymbol{c}_{n}$ and $\boldsymbol{d}_{n}$ in (4) are calculated from:

$$
\begin{aligned}
& \boldsymbol{a}_{n}=\left[\frac{\boldsymbol{A}^{n} L^{2 n}}{2 n !}\right] \boldsymbol{V}_{i}, \boldsymbol{b}_{n}=-\left[\frac{\boldsymbol{A}^{n} L^{2 n}}{2 n !}\right] \boldsymbol{V}_{j}, \\
& \boldsymbol{c}_{n}=\left[\frac{\boldsymbol{A}^{n} \boldsymbol{z} L^{2 n+1}}{(2 n+1) !}\right] \boldsymbol{J}_{i, j} \text { and } \boldsymbol{d}_{n}=-\left[\frac{\boldsymbol{A}^{n} \boldsymbol{z} L^{2 n+1}}{(2 n+1) !}\right] \boldsymbol{J}_{j, i} . \\
& \begin{aligned}
\text { C) : Elements of coefficient matrix in (5) are obtained from: } \\
\qquad \boldsymbol{h}_{0}=\boldsymbol{a}_{0}+\boldsymbol{b}_{0}-\boldsymbol{d}_{0}+\boldsymbol{b}_{1}-\boldsymbol{d}_{1}+\boldsymbol{b}_{2}, \\
\boldsymbol{h}_{1}=-\boldsymbol{c}_{0}+\boldsymbol{d}_{0}-2 \boldsymbol{b}_{1}+3 \boldsymbol{d}_{1}-4 \boldsymbol{b}_{2}, \\
\boldsymbol{h}_{2}=\boldsymbol{a}_{1}+\boldsymbol{b}_{1}-3 \boldsymbol{d}_{1}+6 \boldsymbol{b}_{2}, \\
\boldsymbol{h}_{3}=-\boldsymbol{c}_{1}+\boldsymbol{d}_{1}-4 \boldsymbol{b}_{2}, \\
\boldsymbol{h}_{4}=\boldsymbol{a}_{2}+\boldsymbol{b}_{2} .
\end{aligned}
\end{aligned}
$$

In fact, the complete equation set would have a form of $\sum_{k=0}^{\infty} \boldsymbol{h}_{k} \alpha^{k}=0$, where the vector $\boldsymbol{h}_{k}$ is obtained from the equation at the bottom of the page. In that formula, $\left(\begin{array}{c}n \\ m\end{array}\right)$ is used to denote the number of $m$-combinations from a given set of $n$ elements.

$$
\boldsymbol{h}_{k}=\left\{\begin{array}{l}
\boldsymbol{a}_{\frac{k}{2}}+\sum_{r=k / 2}^{\infty}\left(\begin{array}{c}
2 r \\
k
\end{array}\right) \boldsymbol{b}_{r}-\sum_{r=k / 2}^{\infty}\left(\begin{array}{c}
2 r+1 \\
k
\end{array}\right) \boldsymbol{d}_{r}, \quad \text { if } k \text { is even } \\
-\boldsymbol{c}_{\frac{(k-1)}{2}}-\sum_{r=\frac{(k+1)}{2}}^{\infty}\left(\begin{array}{c}
2 r \\
k
\end{array}\right) \boldsymbol{b}_{r}+\sum_{r=\frac{(k-1)}{2}}^{\infty}\left(\begin{array}{c}
2 r+1 \\
k
\end{array}\right) \boldsymbol{d}_{r}, \text { otherwise }
\end{array}\right.
$$


D) : The per-unit length impedance and admittance matrices of the untransposed transmission lines used to construct the test systems are respectively as follows:

$$
\begin{array}{r}
z=\left[\begin{array}{rrr}
0.0957+0.5253 i & 0.0865+0.2600 i & 0.0840+0.2178 i \\
0.0865+0.2600 i & 0.1002+0.5226 i & 0.0865+0.2600 i \\
0.0840+0.2178 i & 0.0865+0.2600 i & 0.0957+0.5253 i
\end{array}\right] \\
\boldsymbol{y}=\left[\begin{array}{llr}
0.0100+3.4817 i & 0.0000-0.7247 i & 0.0000-0.2337 i \\
0.0000-0.7247 i & 0.0100+3.6881 i & 0.0000-0.7247 i \\
0.0000-0.2337 i & 0.0000-0.7247 i & 0.0100+3.4817 i
\end{array}\right]
\end{array}
$$$$
(\mu S / k m)
$$

The line lengths in the untransposed 39-bus test system are set so that if they are ideally transposed, their impedance magnitudes would be equal to those of the corresponding lines in the original 39-bus test system [42].

E) : In the proposed fault location algorithm, the following steps should be followed for every suspected line:

1- solution of system of (15),

2- calculation of voltage synchrophasors at the faulted line ends from (16),

3- calculation of sum of squared residuals (17),

4- solution of system of (5).

The solution of (15) would be $\boldsymbol{X}=\boldsymbol{G} \boldsymbol{M}$ where $\boldsymbol{G}=\left[\boldsymbol{H}^{\mathrm{T}} \boldsymbol{H}\right]^{-1} \boldsymbol{H}^{\mathrm{T}}$. The coefficient matrix $\boldsymbol{H}$ and thus $\boldsymbol{G}$ is constant and would be calculated and stored offline. Therefore, step 1 turns to $6 \times N_{s}$ multiplications. In step 2, voltage synchrophasors at the faulted line terminals are calculated from (16) by execution of 36 multiplications. In step 3, after calculation of $\boldsymbol{H} \boldsymbol{X}$ by $6 \times N_{s}$ multiplications, sum of squared residuals could be obtained from $[\boldsymbol{M}-\boldsymbol{H} \boldsymbol{X}]^{\mathrm{T}} \times[\boldsymbol{M}-\boldsymbol{H} \boldsymbol{X}]$ by executing $N_{s}$ more multiplications. Step 4 might take up to tens of ms depending on the solver being used. It should be noted that fault location is carried out in the offline stage, and contrary to the algorithms deployed in protective relays, it does not need to be extremely fast.

Overall, for a network with $N_{L}$ lines and $N_{s}$ synchrophasor measurements, the maximum time needed for applying the proposed wide-area fault location is calculated from:

$$
t_{\text {total }}=N_{L}\left(T_{p}+\left(13 N_{s}+36\right) T_{m}\right),
$$

where $T_{p}$ is the time for solving (5), and $T_{m}$ is the execution time of a single complex multiplication. It must be added that (A-1) gives an upper boundary for the total computational time, since it does not consider the possibility of any parallelization on the software and/or hardware level. In the proposed framework, fault location on each line is completely independent of fault location on the other lines. Therefore, the faulted line identification process, i.e., the execution of the above four steps for all suspected lines, could be highly parallelized. This would reduce the total computational time of the whole fault location algorithm to $T_{p}+\left(13 N_{s}+36\right) T_{m}$. Being around several ms, this time is also the same time needed for pinpointing the fault using the proposed method, when the faulted line is known a-priori.

$F)$ : The impedances of the two-terminal test system are as below:

$$
Z_{1}^{S}=Z_{1}^{R}=59 \angle 89^{\circ}(\Omega), Z_{0}^{S}=Z_{0}^{R}=120 \angle 84^{\circ}(\Omega),
$$

Besides, the three-phase voltages of the two sources are given below in p.u.

$V_{a}^{S}=1 \angle 0^{\circ}, V_{b}^{S}=1.01 \angle-120^{\circ}, V_{c}^{S}=1.015 \angle 119^{\circ}$, $V_{a}^{R}=1.015 \angle-12^{\circ}, V_{b}^{R}=1.02 \angle-133^{\circ}, V_{c}^{R}=1.025 \angle 108^{\circ}$.

\section{ACKNOWLEDGMENT}

The first author would like to thank Dr. Ahmad Salehi Dobakhshari of University of Guilan, for his constructive suggestions about the paper. He is also very grateful to the members of Distributed Electrical Systems Laboratory (DESL) at the Swiss Federal Institute of Technology of Lausanne (EPFL) for their professional support during the course of this research.

\section{REFERENCES}

[1] M. M. Saha, J. J. Izykowski, and E. Rosolowski, Fault Location on Power Networks, 1st ed. London, U.K.: Springer, 2010.

[2] IEEE Guide for Determining Fault Location on AC Transmission and Distribution Lines, IEEE Std. C37.114-2004, 2005.

[3] B. Mahamdedi, M. Sanaye-Pasand, S. Azizi, and J. G. Zhu, "Unsynchronised fault-location technique for three-terminal lines," IET Gen., Transm., Distrib., vol. 9, no. 15, pp. 2099-2107, Jun. 2015.

[4] A. T. Johns and S. Jamali, "Accurate fault location technique for power transmission lines," Proc. IEE Gen., Transm., Distrib., vol. 137, no. 6, pp. 395-402, Nov. 1990.

[5] A. A. Girgis, D. G. Hart, and W. Peterson, "A new fault location technique for two- and three-terminal lines," IEEE Trans. Power Del., vol. 7, no. 1, pp. 98-107, Jan. 1992

[6] J. Izykowski, E. Rosolowski, M. M. Saha, M. Fulczyk, and P. Balcerek, "A fault-location method for application with current differential relays of three-terminal lines," IEEE Trans. Power Del., vol. 22, no. 4, pp. 2099-2106, Oct. 2007.

[7] I. Zamora, J. F. Minambres, A. J. Mazon, R. Alvarez-Isasi, and J. Lazaro, "Fault location on two-terminal transmission lines based on voltages," Proc. IEE Gen., Transm., Distrib., vol. 143, no. 1, pp. 1-6, Jan. 1996.

[8] J. Minambres, I. Zamora, A. Mazon, M. Zorrozua, and R. AlvarezIsasi, "A new technique, based on voltages, for fault location on threeterminal transmission lines," Elect. Power Syst. Res., vol. 37, no. 2, pp. 143-151, May 1996.

[9] F. H. Magnago and A. Abur, "Fault location using wavelets," IEEE Trans. Power Del., vol. 13, no. 4, pp. 1475-1480, Oct. 1998.

[10] A. Sharafi, M. Sanaye-Pasand, and P. Jafarian, "Ultra-high-speed protection of parallel transmission lines using current travelling waves," IET Gen., Transm., Distrib., vol. 5, no. 6, pp. 656-666, Jun. 2011.

[11] P. Jafarian and M. Sanaye-Pasand, "A traveling-wave-based protection technique using wavelet/PCA analysis," IEEE Trans. Power Del., vol. 25, no. 2, pp. 588-599, Apr. 2010.

[12] C. Y. Evrenosoglu and A. Abur, "Travelling wave based fault location for teed circuits," IEEE Trans. Power Del., vol. 20, no. 2, pp. 1115-1121, Apr. 2005.

[13] R. Razzaghi, G. Lugrin, H. Manesh, C. Romero, M. Paolone, and F. Rachidi, "An efficient method based on the electromagnetic time reversal to locate faults in power networks," IEEE Trans. Power Del., vol. 28 , no. 3, pp. 1663-1672, Jul. 2013.

[14] A. Borghetti, M. Bosetti, M. D. Silvestro, C. A. Nucci, and M. Paolone, "Continuous-wavelet transform for fault location in distribution power networks: Definition of mother wavelets inferred from fault originated transients," IEEE Trans. Power Syst., vol. 23, no. 2, pp. 380-388, May 2008.

[15] A. J. Mazon, I. Zamora, J. Gracia, K. J. Sagastebeitia, and J. R. Saenz, "Selecting ANN structures to find transmission faults," IEEE Comput. Appl. Power, vol. 14, no. 3, pp. 44-48, Jul. 2001.

[16] Chen and J. C. Maun, "Artificial neural network approach to singleended fault locator for transmission lines," IEEE Trans. Power Syst., vol. 15 , no. 1 , pp. $370-375$, Feb. 2000.

[17] R. Salat and S. Osowski, "Accurate fault location in the power transmission line using support vector machine approach," IEEE Trans. Power Syst., vol. 19, no. 2, pp. 979-986, May 2004. 
[18] A. J. Mazon, I. Zamora, J. F. Minambres, M. A. Zorrozua, J. J. Barandiaran, and K. Sagastebeitia, "A new approach to fault location in two-terminal transmission lines using artificial neural networks," Elect. Power Syst. Res., vol. 56, pp. 261-266, Dec. 2000.

[19] P. Jarventausta, P. Verho, and J. Partanen, "Using fuzzy sets to model the uncertainty in the fault location process of distribution networks," IEEE Trans. Power Del., vol. 9, no. 2, pp. 954-960, Apr. 1994.

[20] M. R. Jegarluei, A. S. Dobakhshari, A. M. Ranjbar, and A. Tayebi, "A new algorithm for fault location on transmission lines by optimal PMU placement," Int. Trans. Elect. Energy Syst., Jul. 2014.

[21] K. P. Lien, C. W. Liu, C. S. Yu, and J. A. Jiang, "Transmission network fault location observability with minimal PMU placement," IEEE Trans. Power Del., vol. 21, no. 3, pp. 1128-1136, Jul. 2006.

[22] J. Zare, F. Aminifar, and M. Sanaye-Pasand, "Synchrophasor-based wide-area backup protection scheme with data requirement analysis," IEEE Trans. Smart Grid, vol. 30, no. 3, pp. 1410-1419, Mar. 2015.

[23] M. Kezunovic and Y. Liao, "Fault location estimation based on matching the simulated and recorded waveforms using genetic algorithms," in Proc. Int. Conf. Develop. Power Syst. Protect., RAI, Amsterdam, The Netherlands, Apr. 2001

[24] A. S. Dobakhshari and A. M. Ranjbar, "A circuit approach to fault diagnosis in power systems by wide-area measurement system," Int. Trans. Elect. Energy Syst., vol. 23, no. 8, pp. 1272-1288, Nov. 2013.

[25] Z. Galijasevic and A. Abur, "Fault location using voltage measurements," IEEE Trans. Power Del., vol. 17, no. 2, pp. 441-445, Apr. 2002.

[26] A. S. Dobakhshari and A. M. Ranjbar, "Application of synchronized phasor measurements to wide-area fault diagnosis and location," IET Gen., Transm., Distrib., vol. 8, no. 4, pp. 716-729, Apr. 2014.

[27] Y. Liao, "Fault location for single-circuit line based on bus-impedance matrix utilizing voltage measurements," IEEE Trans. Power Del., vol. 23, no. 2, pp. 609-617, Apr. 2008.

[28] N. Kang and Y. Liao, "Double-circuit transmission-line fault location with the availability of limited voltage measurements," IEEE Trans. Power Del., vol. 27, no. 1, pp. 325-336, Jan. 2012.

[29] N. Kang and Y. Liao, "Double-circuit transmission-line fault location utilizing synchronized current phasors," IEEE Trans. Power Del., vol. 28, no. 2, pp. 1040-1047, Apr. 2013.

[30] Q. Jiang, X. Li, B. Wang, and H. Wang, "PMU-based fault location using voltage measurements in large transmission networks," IEEE Trans. Power Del., vol. 27, no. 3, pp. 1644-1652, Jul. 2012.

[31] S. Azizi and M. Sanaye-Pasand, "A straightforward method for widearea fault location on transmission networks," IEEE Trans. Power Del., vol. 30, no. 1, pp. 441-445, Feb. 2015.

[32] A. S. Dobakhshari and A. M. Ranjbar, "A novel method for fault location of transmission lines by wide-area voltage measurements considering measurement errors," IEEE Trans. Smart Grid, vol. 6, no. 2, pp. 874-884, Mar. 2015.

[33] G. Feng and A. Abur, "Identification of faults using sparse optimization," in Proc. 52nd Annu. Allerton Conf. Communication, Control, and Computing, Sep. 30-Oct 3., 2014.

[34] S. Azizi, M. Sanaye-Pasand, M. Abedini, and A. Hassani, "A travelingwave-based methodology for wide-area fault location in multiterminal DC systems," IEEE Trans. Power Del., vol. 29, no. 6, pp. 2552-2560, Aug. 2014.

[35] M. Korkali and A. Abur, "Optimal deployment of wide-area synchronized measurements for fault-location observability," IEEE Trans. Power Syst., vol. 28, no. 1, pp. 482-489, Feb. 2013.

[36] S. Azizi, S. Afsharnia, and M. Sanaye-Pasand, "Fault location on multiterminal DC systems using synchronized current measurements," Int. J. Elect. Power Energy Syst., vol. 63, pp. 779-786, Dec. 2014.

[37] N. D. Tleis, Power Systems Modelling and Fault Analysis: Theory and Practice, 7th ed. Oxford, U.K.: Newnes, 2008.

[38] C. A. Apostolopoulos and G. N. Korres, "A novel fault-location algorithm for double-circuit transmission lines without utilizing line parameters," IEEE Trans. Power Del., vol. 26, no. 3, pp. 1467-1478, Jul. 2011.

[39] C. S. Chen, C. W. Liu, and J. A. Jiang, "A new adaptive PMU based protection scheme for transposed/untransposed parallel transmission lines," IEEE Trans. Power Del., vol. 17, no. 2, pp. 395-404, Apr. 2002.

[40] J. C. Li and Y. P. Wu, "A distributed circuit model for three-phase transposed and untransposed transmission lines.," Elect. Power Syst. Res., vol. 19, no. 6, pp. 187-194, Oct. 1990.

[41] C. L. Fortescue, "Method of symmetrical coordinates applied to the solution of polyphase networks," AIEE Trans., vol. 37, no. 2, pp. 1027-1140, Jun. 1918.
[42] M. A. Pai, Energy Function Analysis for Power System Stability. Boston, MA, USA: Kluwer, 1989.

[43] PSCAD/EMTDC, Ver. 4.2.1 Manitoba HVDC Research Centre, 2006.

[44] DIgSILENT Power Factory, Version 14, DIgSILENT GmbH, 2008.

[45] J. Izykowski, Fault Location on Power Transmission Lines. Wroclaw, Poland: Tech. Univ. Wroclaw Press, 2008.

[46] D. A. G. Vieira, D. B. Oliveira, and A. C. Lisboa, "A closed-form solution for untransposed transmission-lines fault location with nonsynchronized terminals," IEEE Trans. Power Del., vol. 28, no. 1, pp. 524-525, Jan. 2013.

[47] C. A. Apostolopoulos and G. N. Korres, "A novel algorithm for locating faults on transposed/untransposed transmission lines without utilizing line parameters," IEEE Trans. Power Del., vol. 25, no. 4, Oct. 2010.

[48] S. Azizi, A. S. Dobakhshari, S. A. N. Sarmadi, and A. M. Ranjbar, "Optimal PMU placement by an equivalent linear formulation for exhaustive search," IEEE Trans. Smart Grid, vol. 3, no. 1, pp. 174-182, Mar. 2012.

[49] B. Xu and A. Abur, "Observability analysis and measurement placement for system with PMUs," in Proc. IEEE Power Syst. Conf. Expo., Oct. 2004, vol. 2, pp. 943-946.

[50] A. Abur and A. G. Exposito, Power System State Estimation: Theory and Implementation. New York, NY, USA: Marcel Dekker, 2004.

[51] P. M. Anderson, Power System Protection. New York, NY, USA: IEEE Press, 1999.

[52] E. Caro, R. Singh, B. C. Pal, A. J. Conejo, and R. A. Jabr, "Participation factor approach for phasor measurement unit placement in power system state estimation," IET Gen., Transm., Distrib., vol. 6, no. 9, Sep. 2012.

[53] IEEE Standard for Synchrophasors for Power Systems, IEEE Std. C37. $118-1,2011$

Sadegh Azizi (S'12) received the B.Sc. degree in electrical engineering from K. N. Toosi University of Technology, Tehran, Iran, in 2007, the M.Sc. degree in electrical engineering from Sharif University of Technology, Tehran, in 2010, and is currently working toward the Ph.D. degree in electrical engineering at the University of Tehran, Tehran.

Currently, he is a guest researcher with the Distributed Electrical Systems Laboratory (DESL) at the Swiss Federal Institute of Technology of Lausanne (EPFL), where he is also an Exchange Ph.D. Student. His research interests include applications of wide-area monitoring, protection and control systems, digital protective relays, and power system stability studies.

Majid Sanaye-Pasand (M'98-SM'05) received the electrical engineering degree from the University of Tehran, Tehran, Iran, in 1988, and the M.Sc. and $\mathrm{Ph} . \mathrm{D}$. degrees in electrical engineering from the University of Calgary, Calgary, $\mathrm{AB}$, Canada, in 1994 and 1998, respectively.

Currently, he is a Professor at the School of Electrical and Computer Engineering, University of Tehran, where he is also with the Control and Intelligent Processing Center of Excellence. His research interests include power system analysis and control, digital protective relays, and application of artificial intelligence.

Mario Paolone (M'07-SM'10) was born in Italy in 1973. He received the M.Sc. degree (Hons.) in electrical engineering and the Ph.D. degree in electrical engineering from the University of Bologna, Bologna, Italy, in 1998 and 2002, respectively.

In 2005, he was appointed Researcher in Electric Power Systems, University of Bologna, where he was with the Power Systems Laboratory until 2011. In 2010, he received Associate Professor eligibility from the Politecnico di Milano, Milan, Italy. Currently, he is Associate Professor at the Swiss Federal Institute of Technology of Lausanne (EPFL), Lausanne, Switzerland, where he accepted the EOS Holding Chair of Distributed Electrical Systems Laboratory.

Dr. Paolone is Secretary and Member of several IEEE and CIGRÉ working groups. He was Co-Chairperson of the technical committee of the ninth edition of the International Conference of Power Systems Transients. His research interests are in the areas of smart grids, with particular reference to real-time monitoring and operation, power system protections, power systems dynamics, and power system transients with particular reference to Lightning-Electromagnetic Pulse (LEMP) interaction with electrical networks. 